\title{
Severe neutropenia secondary to piperacillin/tazobactam therapy
}

Piperacillin-tazobactam has a wide spectrum of activity that includes gram-positive organisms such as staphylococci and streptococci as well as many gram-negative aerobic and anaerobic bacteria. It's known adverse effects include hypersensitivity reactions, neurotoxicity, hepatotoxicity, diarrhea, electrolyte and acid-base disturbances, bleeding disorders, thrombocytopenia, rarely neutropenia and hemolytic anemia.

Although neutropenia is an uncommon side effect of piperacillin-tazobactam, severe life-threatening neutropenia (absolute neutrophil count of zero) is extremely rare, only few cases were reported with such complication.

\section{Case history}

A 50-year-old male patient was admitted to the hospital with a 10-day history of fever, cough and chest pain. He had an unremarkable past medical history. Chest X-ray showed pleural effusion (Fig. 1). Chest CT scan with contrast showed encysted pleural effusion (Fig. 2).

Thoracentesis under ultrasound guidance, revealed exudative effusion; pleural fluid study for AFB, Gram stain, culture and cytology was negative. A tuberculin skin test with 5TU PPD was negative. Sputum for AFB and gram stain was negative. Initial investigations showed a hemoglobin level of $15 \mathrm{~g} / \mathrm{dl}$; total leucocyte (TLC) $15,000 / \mu$ l with $60 \%$ neutrophils, 38\%, lymphocytes, 1\% eosinophils and absolute neutrophil count (ANC) 9000/ $\mathrm{ll}$; platelet count was 250,000/ $\mu \mathrm{l}$.

Because of the severity of his illness and high WBC count; antibiotic therapy was initiated. The patient was treated as parapneumonic pleural effusion and piperacillin/tazobactam $4.5 \mathrm{~g}$ every $8 \mathrm{~h}$, IV plus azithromycin $500 \mathrm{mg}$, p.o. per day for 3 days were initiated empirically. Paracetamol tablets were given on PRN bases. The CBC count was ordered twice per week.

Ten days later the patient did not improve. He continued to have fever in spite of antibiotics. A chest tube was inserted, followed by the administration of intrapleural streptokinase for 3 days. On the following days the fever subsided and the patient was kept on the same antibiotic, while paracetamol was stopped. Sixteen days after admission the absolute neutrophil count dropped to 2000/ $\mu$ l. Thus, CBC count was ordered daily to monitor the WBC count.

On the $20^{\text {th }}$ day, the patient developed leucopenia, the total leucocyte count (TLC) was $900 / \mu$ l with $0 \%$ neutrophils, $95 \%$, lymphocytes and 5\% eosinophils; absolute neutrophil count (ANC) was zero (Fig. 3), while hemoglobin and platelets remain unchanged. The blood cell count was checked by automated blood cell counter and then rechecked manually.
Figure 1. Chest X-ray showed pleural effusion

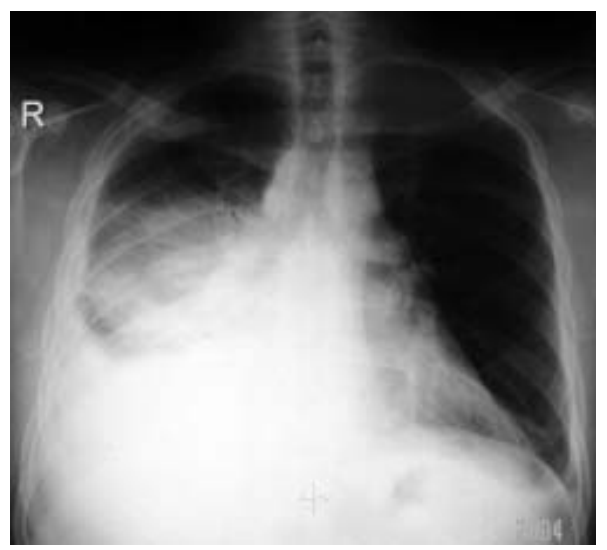

Figure 2. CT scan with contrast showed encysted pleural effusion

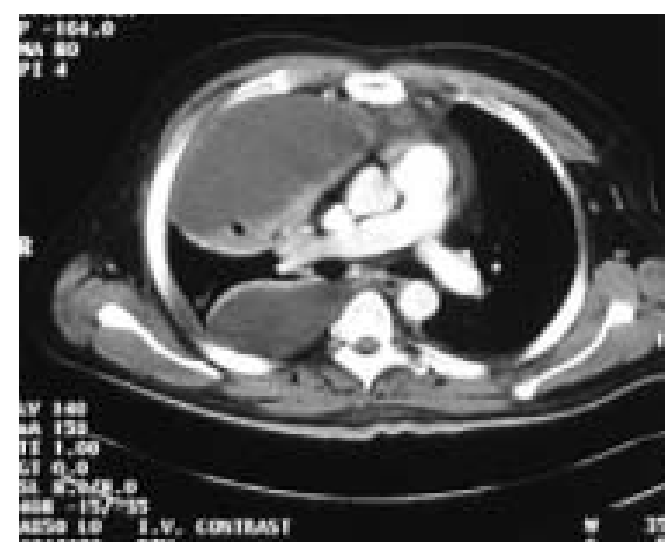

Figure 3. Absolute neutrophil count during the course of the disease

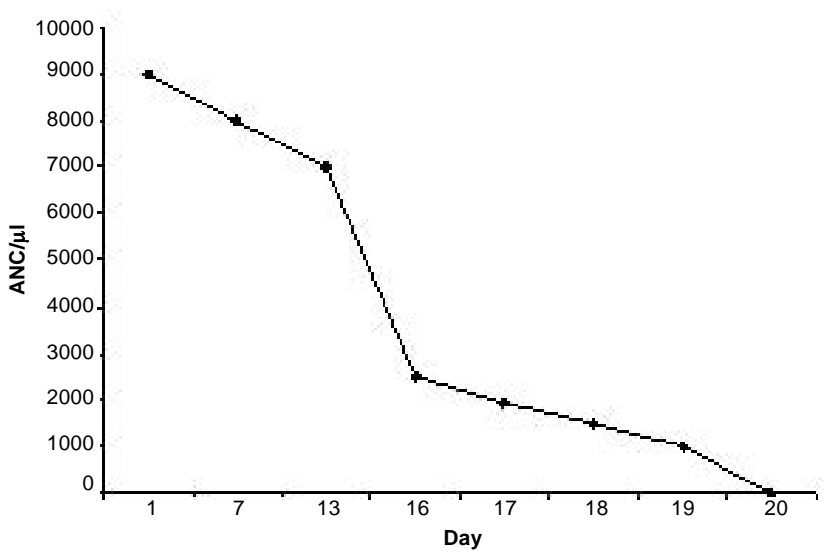


Suspecting drug-induced bone marrow suppression; piperacillin/tazobactam was stopped on the same day and the patient was kept in reverse isolation room. Bone marrow examination revealed a maturation arrest of granulocytic cells. Other marrow components were normal. Neupogen (Filgrastim) Granulocyte stimulating factor (G-CSF) was started at $300 \mu \mathrm{g}$ per day subcutaneously for 3 days. On the next day, the neutrophil count started to rise gradually until it reached $9000 / \mathrm{ml}$ after 4 days. The patient remained afebrile and consequently he was discharged.

\section{Discussion}

Piperacillin/tazobactam is a $\beta$-lactam/ $\beta$-lactamase inhibitor combination with a broad spectrum of antibacterial activity against most gram-positive and gram-negative aerobic bacteria and anaerobic bacteria. Piperacillin/tazobactam is effective and well tolerated in patients with lower respiratory tract infections, intra-abdominal infections, skin and soft tissue infections and febrile neutropenia. In comparative clinical trials against various other antibacterial regimens, piperacillin/ tazobactam has shown higher clinical success rates, particularly in the treatment of patients with intra-abdominal infections and febrile neutropenia. ${ }^{11]}$

Combining tazobactam, a $\beta$-lactamase inhibitor, with the ureidopenicillin, piperacillin, successfully restores the activity of piperacillin against $\beta$-lactamase-producing bacteria. Tazobactam has inhibitory activity, and therefore protects piperacillin against Richmond and Sykes types II-V $\beta$ lactamases, staphylococcal penicillinase and extendedspectrum $\beta$-lactamases. ${ }^{|2|}$

It's known adverse effects include hypersensitivity reactions, neurotoxicity, hepatotoxicity, diarrhea, electrolyte and acid-base disturbances, bleeding disorders, neutropenia and thrombocytopenia and rarely hemolytic anemia. ${ }^{[3]}$

Leucopenia is an uncommon but serious adverse effect of piperacillin and other $\beta$-lactam antibiotics. There have been several previous reports of leucopenia and bone marrow suppression following the use of piperacillin ${ }^{[4|,| 5 \mid}$ and piperacillin/tazobactam. ${ }^{[6-|8|}$

This bone marrow suppression is usually reversible, recovers with discontinuation of the drug and is possibly related to direct toxicity to myeloid precursors..$^{|9|}$ Large cumulative doses are needed and neutropenia rarely develops before 10 days of therapy. ${ }^{[9] \mid[10]}$

Our patient developed neutropenia 20 days after the start of piperacillin/tazobactam. In previous reports, neutropenia has been reported to occur 11-17 days after the therapy was begun. ${ }^{|6|, \mid 7]}$ Also bone marrow suppression occurred in patients who had received a cumulative piperacillin/tazobactam dose of $4919 \pm 1975 \mathrm{mg} / \mathrm{kg},{ }^{[6]}$ i.e. $4372 \pm 1755 \mathrm{mg} / \mathrm{kg}$, body weight of piperacillin. Our patient had received piperacillin/ tazobactam in a dose of $13.5 \mathrm{~g} /$ day, with a cumulative piperacillin dose of $3000 \mathrm{mg} / \mathrm{kg}$ body weight, which falls within the suppressive range mentioned above.

The transient leucopenia can be caused by streptokinase infusion due to complements activation. ${ }^{[11]}$ In this patient streptokinase was given locally into the pleural space and leucopenia was caused by bone marrow suppression as revealed by bone marrow examination.

The diagnosis of bone marrow suppression due to piperacillin/tazobactam in this patient is supported by many facts: First, the patient was not receiving any medications except piperacillin/tazobactam, when bone marrow suppression was noticed. Second, the neutrophil counts returned towards normal within few days after discontinuation of the antibiotic and initiation of Neupogen (Filgrastim).

Thus, bone marrow suppression especially neutropenia, is a serious adverse effect of piperacillin/tazobactam, which should be kept in mind while treating patients with this drug, especially in patients who received a high cumulative dose.

\section{F. Y. Khan}

Department of Medicine, Hamad General Hospital, P.O. Box 3050, Doha, Qatar E-mail: fakhanqal@yahoo.co.uk

\section{References}

1. Young M, Plosker GL: Piperacillin/tazobactam: a pharmacoeconomic review of its use in moderate to severe bacterial infections. Pharmacoeconomics 2001;19:1135-75.

2. Bryson HM, Brogden RN. Piperacillin/tazobactam. A review of its antibacterial activity, pharmacokinetic properties and therapeutic potential. Drugs 1994;47:506-35.

3. Kaucers A, Crowe SM, Grayson ML, Hoy JF, editors. The use of antibiotics: A clinical review of antibacterial, antifungal and antiviral drugs. Oxford: Butterworth Heinemann 1997.

4. Bressler, RB, Huston DP. Piperacillin-induced anemia and leucopenia. South Med J 1986;79:255-6.

5. Wilson C, Greenhood G, Remington JS, Vosti KL. Neutropenia after consecutive treatment courses with nafcillin and piperacillin. Lancet 1979;1:1150.

6. Reichardt P, Handrick W, Linke A, Schille R, Kiess W. Leukocytopenia, thrombocytopenia and fever related to piperacillin/tazobactam treatment - a retrospective analysis in 38 children with cystic fibrosis. Infection 1999;27:355-6.

7. Ruiz-Irastorza G, Barreiro G, Aguirre C. Reversible bone marrow depression by high-dose piperacillin/tazobactam. Br J Haematol 1996;95:611-2.

8. Gerber L, Wing EJ. Life-threatening neutropenia secondary to piperacillin/ tazobactam therapy. Clin Infec Dis 1995;21:1047-8.

9. Neftel KA, Hauser SP, Müller MR. Inhibition of granulopoiesis in vivo and in vitro by beta-lactam antibiotics. J Infect Dis 1985;152:90-8.

10. Singh N, Yu VL, Mieles LA, Wagener MM. Beta-lactam antibiotic-induced leukopenia in severe hepatic dysfunction: risk factors and implications for dosing in patients with liver disease. Am J Med 1993;94:251-6.

11. Frangi $D$, Gardinali M. Abrupt complement activation and transient neutropenia in patients with acute myocardial infarction treated with streptokinase. Circulation 1994;89:76-80.

\section{Join "IndPharm" \\ IJP uses "IndPharm" to broadcast announcements. Want to join? Please E-mail: adithan@vsnl.com}

